\title{
Vegetation Database of the Colombian Caribbean Region
}

\author{
Jesús Orlando Rangel-Churio \& Edgar Andrés Avella-Muñoz
}

\begin{abstract}
Dry and humid forests around wetlands at the department of Córdoba, Caribbean region of Colombia Fragment forests around swamp complexes in several localities at the department of Córdoba were sampled with 77 plots. In each plot all individuals with height up to $1.5 \mathrm{~m}$ were censused, measuring their diameter at breast height, coverage and individual height. Inside each area, the herbaceous layer was inventoried with 5 plots of $2 \times 2 \mathrm{~m}$. Observations about disturbance (intervention class, grade, matrix and contrast type) were taken and physical-chemical soil conditions were characterized to complement the characterization with basis on floristic composition and structure. Using the Twinspan program (PC Ord ver. 4) global tables of group segregation were obtained with which a group differentiation was found. These groups showed a floristic composition closely related with the patterns observed on the field. Around the swamps, vegetation is grouped in the class Craveto tapieae-Astronietea guianensis, order Bursero simauroubaeCavanillesietalia platanifoliae characterized by the dominance of Cavanillesia platanifolia. In this humid forest formation (26 Lev), characterized by large-size individuals of Cavanillesia platanifolia, are included the association of Cratevo tapiae-Cavanillesion platanifoliae alliance like forests of Cordietum protacto-panamensis, Cappari odoratissimatis-Cavanilletum platanifoliae and the mixed palm Sabali mauritiformis-Cavanillesietum platanifoliae. There are also other plant communities that can be assigned to the dry forest formation $(51 \mathrm{Lev})$ characterized by the dominance of tree or shrub size individuals principally Cochlospermum vitifolium, Apeiba aspera, Trichilia acuminata, Attalea butyraceae and Guazuma ulmifolia. Among the characterized associations are Cinnamomo triplinervis-Apeibetum asperae, Cochlospermo vitifoli-Attaleetum butyraceae, Cordia alliodorae-Attaleetum butyraceae and Adenocalymno inundati-Astronietum graveolentis. In this group, forests around the swamps in flooded and non-flooded areas (related to the tropical dry forest formation) have two formation types, one with vigorous elements, height up to 18 meters as the associations of the Dialio guinensis-Mataybion elegantis alliance that include the mixed palm Viticis capitatae-Acrocomietum aculeatae and the Xilopio aromaticae-Tapiretum guianensis. The Phillanto elsiae-Tabebuion roseae alliance includes low rise vegetation, woods and residual forests, as the Annono punicifoliae-Pithecellobietum lanceolati and the shoreline Symmerio paniculatae-Bactrietum guieenensis mangrove thickets in flooded areas. Another forest and thicket in flooded areas (5 Lev) are the "campanales" of Caseario tremulae-Samaneetum samanensis and the forests of the Coccolobo costatae-Acacietum huilanae association and the thickets dominated by Montrichardia arborescens. The structural arrangement, particularly classes distribution is related with type and degree of perturbation of the characterized communities; logging and grazing distort the disetaneo behaviour of natural forests where the higher' percentage of individuals should be distributed in the first classes. The Caseario tremulae Samaneetum samanensis association is a representative case where the intensive grazing has driven this flooded forest to degradation; this is observable in the height classes' distribution, with the higher individuals' percentage (31\%) in class III. The intervention grade of the humid and dry tropical forests characterized in this study can be classified from low (26\%) to high (13\%) predominating the mid degree $(61 \%)$ making evident the current pressure to which this ecosystems are subjected mainly due to land use change for animal breeding, agriculture or mining.However there are still fragmented forests with acceptable characteristics for conservation which will be a starting point for the formulation of ecological restoration models. This report describes the available content in the Vegetation Database of the Colombian Caribbean Region (GIVD ID SA-CO-002).
\end{abstract}

Keywords: dry forest; humid forest; wetland vegetation.

\section{Vegetation Database of the Colombian Caribbean Region}

Scope: The Colombian Caribbean Region database compiles information about relevés of different vegetation types growing from estuarine landscapes to isolated mountain massifs above 3,000 m.a.s.l., including inventories of all plant species according to their stratification.

Status: emerging Period: 1976-2009

Database manager(s): Jesús Orlando Rangel-Churio (jorangelc@gmail.com); Edgar Andrés Avella-Muñoz (eaavella@yahoo.com)

Owner: Jesús Orlando Rangel-Churio

Web address: [NA]

Availability: not yet available

Database format(s): Excel

Online upload: no

Online search: no

Publication: [NA]

Plot type(s): normal plots

Non-overlapping plots: 320

Export format(s): Excel

Plot-size range: $20-1,200 \mathrm{~m}^{2}$

Estimate of existing plots: 500

Completeness: $64 \%$ 


\begin{tabular}{|c|c|}
\hline Total plot observations: 320 & Number of sources: 12 \\
\hline \multicolumn{2}{|l|}{ Countries: CO: $100.0 \%$} \\
\hline \multicolumn{2}{|l|}{ Forest: $[N A]$ - Non-forest: [NA] } \\
\hline \multicolumn{2}{|c|}{$\begin{array}{l}\text { Guilds: all vascular plants: } 100 \% \text {; bryophytes (terricolous or aquatic): } 100 \% \text {; lichens (terricolous or aquatic): } 100 \% \text {; non-terricolous taxa (epiphytic } \\
\text { saxicolous, lignicolous): } 100 \%\end{array}$} \\
\hline \multicolumn{2}{|c|}{$\begin{array}{l}\text { Environmental data: altitude: } 95 \% \text {; slope aspect: } 50 \% \text {; slope inclination: } 90 \% \text {; soil depth: } 80 \% \text {; surface cover other than plants (open soil, litter, } \\
\text { bare rock etc.): } 30 \% \text {; soil pH: } 40 \% \text {; other soil attributes: } 30 \%\end{array}$} \\
\hline \multicolumn{2}{|c|}{$\begin{array}{l}\text { Performance measure(s): presence/absence only: } 100 \% \text {; cover: } 70 \% \text {; number of individuals: } 60 \% \text {; measurements like diameter or height of } \\
\text { trees: } 60 \%\end{array}$} \\
\hline \multicolumn{2}{|c|}{$\begin{array}{l}\text { Geographic localisation: GPS coordinates (precision } 25 \mathrm{~m} \text { or less): } 20 \% \text {; point coordinates less precise than GPS, up to } 1 \mathrm{~km} \text { : } 60 \% \text {; small grid } \\
\text { (not coarser than } 10 \mathrm{~km}): 10 \% \text {; political units or only on a coarser scale }(>10 \mathrm{~km}): 10 \%\end{array}$} \\
\hline \multicolumn{2}{|c|}{ Sampling periods: 1970-1979: 25.0\%; 1990-1999: 10.0\%; 2000-2009: 65.0\% } \\
\hline & \\
\hline
\end{tabular}

Jesús Orlando Rangel-Churio* (jorangelc@ gmail.com), Edgar Andrés Avella-Muñoz (eaavella@ yahoo.com)

Instituto de Ciencias Naturales, Universidad Nacional de Colombia, Carrera 30 No. 45-03, 111321 Bogota, COLOMBIA

*Corresponding author 